\title{
On the Theory of Wave Propagation Through a Concentrically Stratified Troposphere With a Smooth Profile
}

\author{
H. Bremmer
}

(February 1, 1960)

\section{Part I. Discussion of the Extended W.K.B. Approximation}

The W.K.B. approximation for the solution of the height-gain differential equation for a curved stratified troposphere is discussed in detail. The approximation depends mainly on a variable $u_{l}(r)$ which can be interpreted as the height dependent contribution of the phase for a field solution obtained by separation of variables. An expansion of $u_{l}(r)$ with the aid of partial integrations leads to further approximations which facilitate the determination of the eigenvalues, and of the amplitudes of the modes connected with the propagation problem. The influence of the refractive-index profile, if assumed as smooth, then appears to be restricted to a dependence on the surface values of this index and of its gradient insofar as propagation over the ground is concerned. Further, all height effects of elevated antennas can be expressed in terms of the distance to the corresponding radio horizon. This results in simple relations between the fields connected with two different refractive-index profiles, provided both profiles coincide near the earth's surface.

\section{Introduction}

The propagation theory of a concentrically stratified atmosphere usually concerns a discussion of the corresponding height-gain differential equation. The W.K.B. approximation of the latter has amply been discussed, but little attention has been paid to its corrections. Some material on the form of these corrections has been presented by Pekeris $[1]^{2}$ while applying an earth-flattening approximation. This paper concerns (in part II) an expansion of the solution for a curved stratified atmosphere which starts with an "extended W.K.B. approximation." By the latter we understand a well-known modification of the W.K.B. approximation, in terms of Hankel functions of order $1 / 3$; it is determined such as to remain finite at a turning point of the geometrical optics trajectory associated with the solution. The discussion of this extended W.K.B. approximation (in part I) shows the dominating role of the refractive-index profile near the earth's surface, provided that this profile and all its derivatives are continuous functions of the height throughout the troposphere.

\section{Reduction to a Scalar Problem}

We start from Maxwell's equations for time-harmonic solutions (time factor $e^{-i \omega f}$ ) for a medium with spherical symmetry, the refractive index $n(r)$ of which only depends on the distance $r$ to the center of symmetry. We further assume a permeability 1 ; the equations in question then read as follows in Gaussian cgs units:

$$
\begin{gathered}
\text { Curl } \mathbf{e}-i \frac{\omega}{c} \mathbf{h}=0, \\
\operatorname{Curl} \mathbf{h}+i \frac{\omega}{c} n^{2}(r) \mathbf{e}=0 .
\end{gathered}
$$

${ }^{1}$ Contributions from Philips Research Laboratories, N. V. Philips' Glocilampenfabrieken, Eindhoven, Netherlands.

${ }^{2}$ Figures in brackets indicate the literature references at the end of this paper. 
The two standard solutions, known as the electric and the magnetic solution respectively, can be represented by:

$$
\left.\begin{array}{l}
\mathbf{e}_{e}=\frac{i c}{\omega} \frac{1}{n^{2}(r)} \operatorname{curl} \operatorname{curl}\left\{n(r) \Pi_{e} \mathbf{r}\right\}, \\
\mathbf{h}_{e}=\operatorname{curl}\left\{n(r) \Pi_{e} \mathbf{r}\right\},
\end{array}\right\}
$$

and

$$
\left.\begin{array}{l}
\mathbf{e}_{m}=\frac{\imath \omega}{c n^{2}(r)} \cdot \operatorname{curl}\left\{n^{2}(r) \Pi_{m} \mathbf{r}\right\}, \\
\mathbf{h}_{m}=\operatorname{curl}\left[\frac{1}{n^{2}(r)} \cdot \operatorname{curl}\left\{n^{2}(r) \Pi_{m} \mathbf{r}\right\}\right] .
\end{array}\right\}
$$

The symbol $\mathbf{r}$ represents the radial vector of length $\left(x^{2}+y^{2}+z^{2}\right)^{1 / 2}$.

The Maxwell eq (2) is verified at once for the electric solution (3); the same holds with respect to the other Maxwell eq (1) for the magnetic solution (4). The remaining Maxwell equations can be checked by deriving the following relations with the aid of a tedious analysis based on vector-field identities:

$$
\begin{gathered}
\text { Curl } \mathbf{e}_{e}-\frac{i \omega}{c} \mathbf{h}_{e}=-\frac{i c}{\omega} \cdot \operatorname{curl}\left[\mathbf{r}\left\{\frac{1}{n} \cdot \Delta \Pi+\left(\frac{\omega^{2}}{c^{2}} n-\frac{d^{2}}{d r^{2}} \frac{1}{n}\right) \Pi\right\},\right. \\
\operatorname{Curl} \mathbf{h}_{m}+\frac{i \omega}{c} n^{2} \mathbf{e}_{m}=-\operatorname{curl}\left[\mathbf{r}\left\{\Delta \Pi+\left(\frac{\omega^{2}}{c^{2}} n^{2} \Pi\right\}\right] .\right.
\end{gathered}
$$

Therefore, both Maxwell equations are satisfied, for the electric as well as the magnetic solution, if the scalar $I$ does satisfy a wave equation of the form,

$$
\Delta \Pi+\frac{\omega^{2}}{c^{2}} n_{\mathrm{eff}}^{2}(r) \Pi=0 ;
$$

the effective refractive index then has to be defined as follows:

$$
\begin{array}{r}
n_{\mathrm{eff}}^{2}(r)=n^{2}-\frac{c^{2}}{\omega^{2}} n \cdot \frac{d^{2}}{d r^{2}} \frac{1}{n} \text { for the electric solution, } \\
n_{\mathrm{eff}}^{2}(r)=n^{2} \quad \text { for the magnetic solution. }
\end{array}
$$

\section{Height-Gain Differential Equation and Its Extended W.K.B. Approximation}

We are particularly interested in the solutions corresponding to a vertical electric or magnetic dipole. In the system of spherical coordinates $r, \theta, \varphi\left(x+i y=r \sin \theta e^{i \varphi}, z=r \cos \theta\right)$ this dipole may be situated at $r=b, \theta=0$. The field then becomes independent of $\varphi$, and particular solutions $\Pi_{l}$ of $(5)$ are found by a separation of variables according to

$$
\Pi_{l}=f_{l}(r) P_{l}(\cos \theta) .
$$

The corresponding height-gain differential equation for $f_{l}(r)$ can be put in the form

$$
\left\{\frac{d^{2}}{d r^{2}}+\frac{\omega^{2}}{c^{2}} m_{l}^{2}(r)\right\}\left\{r f_{l}(r)\right\}=0,
$$

if

$$
m_{l}^{2}(r) \equiv n_{\mathrm{eff}}^{2}(r)-\frac{l(l+1)}{k_{0}^{2} r^{2}}
$$

$k_{0}=\omega / c$ being the wave number in vacuum.

The W.K.B. approximation of (8) consists of a linear combination of the functions

$$
\frac{e^{ \pm i k_{0}} \int^{\tau} m_{l}(s) d s}{\left\{m_{l}(r)\right\}^{1 / 2}} ;
$$


it therefore breaks down at a so-called turning point, that is a zero $r=r_{l}$ of $m_{l}(r)$. In the case of a single turning point a better approximation is obtained, as is well known, by approximating $m_{l}^{2}(r)$ near $r=r_{l}$ by a linear profile; the equation can then be solved there rigorously with the aid of Hankel functions of order $1 / 3$. This procedure leads to the following "extended W.K.B. approximation:"

$$
r f_{l}(r) \sim A_{l} \frac{\left\{\int_{t_{l}}^{r} m l(s) d s\right\}^{1 / 2}}{\left\{m_{l}(r)\right\}^{1 / 2}} H_{1 / 3}^{(1)}\left\{k_{0} \int_{r_{l}}^{r} m_{l}(s) d s\right\} ;
$$

it reduces to the conventional W.K.B. approximation if the Hankel function is replaced by the first term of its asymptotic expansion for large arguments.

In (10) we assume $\operatorname{Im} m_{l} \geq 0$, which guarantees an exponential decrease for $r \rightarrow \infty$ if $\operatorname{Im} m_{l}$ differs from zero; the function (10) then represents an approximation in accordance with the radiation condition at infinity. In part II of this paper (10) will appear as the first term of a complete expansion for the solution $\Pi_{l}$.

\section{Position of a Turning Point for a General Profile of the Refractive Index}

The significance of a turning-point level $r=r_{l}$ is obvious when $r_{l}$ is a real quantity larger than the earth's radius $a$. We then consider the relation

$$
n_{\mathrm{eff}}(r) \cdot r \cdot \sin \tau(r)=\frac{\{l(l+1)\}^{1 / 2}}{k_{0}}
$$

it determines a ray trajectory in a stratified troposphere with refractive index $n_{\text {eff }}$, if $\tau(r)$ represents the angle, at any level $r$, between the tangent to the trajectory and the radius vector towards the center of the earth. On the other hand, the turning-point relation $m_{l}\left(r_{l}\right)=0$ can be written as follows in view of $(9)$ :

$$
n_{\mathrm{eff}}\left(r_{l}\right) \cdot r_{l}=\frac{\{l(l+1)\}^{1 / 2}}{k_{0}}
$$

Therefore, $r=r_{l}$ characterizes the level at which the trajectory in question becomes horizontal $(\tau=\pi / 2)$. In other words, the turning-point level constitutes an altitude at which this trajectory passes continuously from a rising branch into a descending branch.

In the absence of a turning point in the physical space $a<r<\infty$, a zero $r_{l}$ of $m_{l}(r)$ might occur in the interval $r<a$ outside the troposphere (in accordance with some extrapolation there of $n(r)$ ), or also at some complex value of $r$. The "extended W.K.B. approximation" (10), defined with the aid of such a nonphysical turning-point level, will still be important when the latter proves to be situated near the section $a<r<\infty$ of the real axis in the complex $r$-plane.

As a matter of fact zeros $r_{l}$ of this type occur for the most important modes (7), since all these modes correspond to complex $l$ values situated near $l_{0}$ defined by

$$
\frac{\omega}{c} a n_{\mathrm{eff}}(a)=\left\{l_{0}\left(l_{0}+1\right)\right\}^{1 / 2}
$$

According to (12) $l_{0}$ itself is connected with a ray trajectory tangenting the earth's surface, $r_{l}$ then assuming the value $a$. Hence we expect for all eigenvalues $l$ near $l_{0}$ (to be discussed in part II) a corresponding turning point $r_{l}$ near $a$.

The exact position of this turning point can be derived from an expansion to be obtained as follows. We replace (12) by the equivalent equation:

$$
M_{\mathrm{eff}}^{2}\left(r_{l}\right)=\frac{l(l+1)}{k_{0}^{2} a^{2}}=C_{l}^{2}, \text { say, }
$$


in which

$$
M_{\text {eff }}(r)=\frac{r}{a} \cdot n_{\text {eff }}(r)
$$

denotes a modified refractive index which includes the effects of both the curvature and the profile of the effective refractive index $n_{\text {eff }}$. We consider the following Taylor expansion of (14):

$$
M_{\mathrm{eff}}^{2}(a)+\sum_{j=1}^{\infty} \frac{\Lambda_{-j}}{a^{j} j !}\left(r_{l}-a\right)^{j}=\frac{l(l+1)}{k_{0}^{2} a^{2}},
$$

in which the dimensionless parameters

$$
\Lambda_{j} \equiv a^{j}\left\{\frac{d^{j}\left(M_{\mathrm{eff}}^{2}\right)}{d r^{j}}\right\}_{\tau=a}
$$

characterize the complete tropospheric profile. In view of (14), (15), and (9) we may replace (16) by

$$
\sum_{i=1}^{\infty} \frac{\Lambda_{j}}{a^{j} j !}\left(r_{l}-a\right)^{j}=C_{l}^{2}-n_{\mathrm{eff}}^{2}(a)=-m_{l}^{2}(a) .
$$

We next assume the possibility of inverting this latter relation. The inverted series can be represented as follows:

$$
\frac{r_{l}-a}{a}=-\frac{m_{l}^{2}(a)}{\Lambda_{1}}-\frac{\Lambda_{2}}{2 \Lambda_{1}^{3}} \cdot m_{l}^{4}(a)+\left(\frac{\Lambda_{3}}{6 \Lambda_{1}^{4}}-\frac{\Lambda_{2}^{2}}{2 \Lambda_{1}^{5}}\right) \cdot m_{l}^{6}(a)+\left(\frac{5}{12} \frac{\Lambda_{2} \Lambda_{3}}{\Lambda_{1}^{6}}-\frac{5}{8} \frac{\Lambda_{2}^{3}}{\Lambda_{1}^{7}}-\frac{\Lambda_{4}}{24 \Lambda_{1}^{5}}\right) \cdot m_{l}^{8}(a)+\cdots
$$

It shows how the turning point can easily be evaluated for modes with small values of $m_{l}^{2}(a)=$ $n_{\mathrm{eff}}^{2}(a)-C_{l}^{2}$, which, however, are the only modes of practical interest.

\section{Dependence of the Extended W.K.B. Approximation on the Profile}

In view of (10) this approximation can be represented by the expression

$$
r f_{l}(r) \sim A_{l} \frac{\left\{u_{l}(r)\right\}^{1 / 2}}{\left\{\frac{d}{d r} u_{l}(r)\right\}^{1 / 2} \cdot H_{\frac{1}{3}}^{(1)}\left\{u_{l}(r)\right\},}
$$

which depends uniquely on the variable:

$$
u_{l}(r)=k_{0} \int_{r_{l}}^{r} m_{l}(s) d s=k_{0} a \int_{r_{l}}^{r} \frac{d s}{s}\left\{\boldsymbol{M}_{\mathrm{eff}}^{2}(s)-C_{l}^{2}\right\}^{1 / 2} .
$$

This variable constitutes, apart from the factor $i$, the exponent in the asymptotic approximation of (19) for large values of $\left|u_{l}(r)\right|$. Therefore, $u_{l}$ may be interpreted as the radial contribution to the phase of the complete wave function $f_{l}(r) P_{l}(\cos \theta)$.

Unfortunately, the definition (20) of $u_{l}$ involves the turning point $r_{l}$; hence, the expansion (18) should be substituted in order to show the complete dependence of $u_{l}(r)$ on the profile $M_{\text {eff }}(r)$. Under practical circumstances, however, this dependence can also be established without having to resort to the turning point at all. In fact, by applying partial integrations to (20) we obtain an expansion in which the turning point disappears in all but the last term, such in consequence of (14). The expansion in question reads:

$$
\begin{aligned}
u_{l}(r)=k_{0} a & {\left[\sum _ { j = 0 } ^ { p - 1 } \frac { 2 } { 3 } \frac { 2 } { 5 } \frac { 2 } { 7 } \cdots \frac { 2 } { ( 2 j + 3 ) } ( - 1 ) ^ { j } \{ M _ { \mathrm { eff } } ^ { 2 } ( r ) - C _ { l } ^ { 2 } \} ^ { j + 3 / 2 } \cdot \frac { d ^ { j } } { d ( M _ { \mathrm { eff } } ^ { 2 } ) ^ { j } } \left\{\frac{1}{\left.r \frac{d M_{\mathrm{eff}}^{2}}{d r}\right\}}\right.\right.} \\
& +\frac{2}{3} \frac{2}{5} \frac{2}{7} \cdots \frac{2}{(2 p+1)}(-1)^{p} \int_{s=r_{l}}^{s=r}\left\{M_{\mathrm{eff}}^{2}(s)-C_{l}^{2}\right\}^{p+1 / 2} \cdot \frac{d^{p}}{d\left\{M_{\mathrm{eff}}^{2}(s)\right\}^{p}}\left\{\frac{1}{\left.\left.s \frac{d\left(M_{\mathrm{eff}}^{2}\right)}{d s}\right\} d M_{\mathrm{eff}}^{2}(s)\right] .}\right.
\end{aligned}
$$


This expression suggests the infinite series

$$
u_{l}(r)=k_{0} a \sum_{j=0}^{\infty} \frac{2}{3} \frac{2}{5} \frac{2}{7} \cdots \frac{2}{(2 j+3)}(-1)^{j}\left\{M_{\mathrm{eff}}^{2}(r)-C_{l}^{2}\right\}^{j+3 / 2} \cdot \frac{d^{j}}{d\left\{M_{\mathrm{eff}}^{2}(r)\right\}^{j}}\left\{\frac{1}{r \frac{d M_{\mathrm{eff}}^{2}}{d r}}\right\} .
$$

In the case of convergence of this series, we can verify, differentiating term by term, the relation

$$
\frac{d u_{l}(r)}{d r}=\frac{k_{0} a}{r}\left\{M_{\mathrm{eff}}^{2}(r)-C_{l}^{2}\right\}^{1 / 2}
$$

this proves the correctness of (21) when taking into account the property $u_{l}\left(r_{l}\right)=0$.

As an example we consider the magnetic solution in the case of the "Eckersley profile." The refractive index $n(r)$ of the latter is defined by

$$
n^{2}(r)=n^{2}(a)\left\{\frac{a}{a_{\mathrm{eff}}}+\left(1-\frac{a}{a_{\mathrm{eff}}}\right) \frac{a^{2}}{r^{2}}\right\}
$$

$a_{\text {eff }}$ being the effective earth radius. The corresponding effective modified refractive index, viz

$$
M_{\mathrm{eff}}(r)=n(a)\left\{\frac{r^{2}}{a \cdot a_{\mathrm{eff}}}+\left(1-\frac{a}{a_{\mathrm{eff}}}\right)\right\}^{\frac{1}{2}}
$$

leads to the following series for $(21)$ :

$$
\left\{u_{l}(r)\right\}_{\mathrm{Eck}}=\frac{k_{0} a r}{2} \sum_{j=0}^{\infty} j ! \frac{2}{3} \frac{2}{5} \cdots \frac{2}{(2 j+3)}\left\{\frac{a \cdot a_{\mathrm{eff}}}{n^{2}(a)}\right\}^{j+1}\left\{\frac{n^{2}(a)}{a \cdot a_{\mathrm{eff}}}+\frac{n^{2}(a)\left(1-a / a_{\mathrm{eff}}\right)-C_{l}^{2}}{r^{2}}\right\}^{j+3 / 2}
$$

The convergence condition here amounts to:

$$
\left|1+\frac{a \cdot a_{\text {eff }}}{r^{2}}\left\{1-\frac{a}{a_{\text {eff }}}-\frac{C_{l}^{2}}{n^{2}(a)}\right\}\right|<1
$$

in view of the smallness in practice of the quantities $(r-a) / a=h / a$ and $\delta C_{l}=C_{l}-n(a)$ this reduces to the following approximative condition

$$
\left|\frac{2 h}{a}-\frac{2 a_{\mathrm{eff}}}{a} \frac{\delta C_{l}}{n(a)}\right|<1
$$

which is satisfied for all relevant values of the height $h$ and the parameter $C_{l}$. The actual smooth profiles, excluding ducts and so on, deviate only slightly from the Eckersley profile; this suggests the validity of the corresponding expansion (21) in all practical cases.

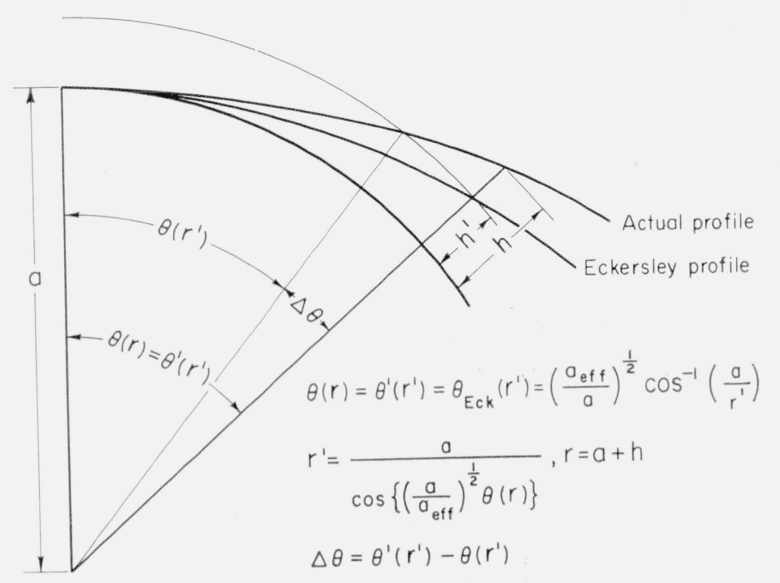

Figure 1. Geometry of radio ray bending. 


\section{Formulation of the Eigenvalue Problem}

It is now well established [2] that our propagation problem concerning the two media of the spherical earth and its surrounding stratified atmosphere can be reduced approximatively to a one-medium problem by introducing a proper boundary condition at the earth's surface. This condition is arrived at by considering the surface impedance

$$
Z=\frac{4 \pi}{c} \frac{E_{\|}}{H_{\|}}
$$

which depends on the ratio of the horizontal components $E_{\|}$and $H_{\|}$of the electric and the magnetic field at the earth's surface. The factor $4 \pi / c$ is such that $Z$ represents the ratio of the tangential electric field at the earth's surface, and of the density of a properly chosen twodimensional current distribution on this surface; the effect of this fictitious current distribution is identical with that of the actual field inside the earth.

The value of $Z$ depends on the field solution under consideration. In the case of a flat boundary, and a plane incident wave arriving from the atmosphere, e.g., $Z$ depends on the direction of arrival $\tau$ of the incident wave. The assumption which enables us to leave out of consideration the medium inside the earth altogether is that $Z$ may be replaced by its special value for a plane wave at grazing incidence $(\tau=\pi / 2)$. The corresponding boundary condition, viz

$$
E_{\|}=\frac{c}{4 \pi} Z_{\tau=\pi / 2} H_{\|} \quad \text { at } r=a,
$$

can be worked out with the aid of (3) and (4) for the electric and magnetic solution respectively. It results in a relation of the following form for the scalar $\Pi$ describing these solutions:

$$
\frac{\partial}{\partial r}(r \Pi)=\frac{\Gamma}{a} r \Pi \quad \text { at } r=a .
$$

The parameter $\Gamma$ is then given, respectively, by

$$
\Gamma_{e}=\frac{i \omega}{4 \pi} a n^{2}(a) Z_{e l}-a \frac{n^{\prime}(a)}{n(a)}=i k_{0} a n(a) \frac{\left(n_{e}^{2}-1\right)^{1 / 2}}{n_{e}^{2}}-a \frac{n^{\prime}(a)}{n(a)} ; \quad \Gamma_{m}=\frac{4 \pi a \omega}{i c^{2} Z_{m}}=-i k_{0} a n(a)\left(n_{e}^{2}-1\right)^{1 / 2} ;
$$

in these expressions $n(a)$ denotes the atmospheric refractive index at the earth's surface, and $n_{e}$ the refractive index associated with a refraction from the atmosphere towards the earth.

The boundary condition (23) can only be fulfilled for a discrete set of modes (7). Henceforth the parameter $l$ will refer to a special complex eigenvalue fixing the order of the Legendre function $P_{l}(\cos \vartheta)$ that constitutes a factor of the field of such a mode. The amplitudes of the modes depend on the source of the field. In the case of a vertical electric or magneticdipole point source at $r=b, \vartheta=0$ the expansion in terms of these modes becomes as follows [3]:

$$
\Pi=\frac{\pi B}{c a^{2} b} \sum_{l} \frac{(2 l+1)}{\frac{\partial}{\partial \lambda}\left\{\frac{\frac{d}{d r}\left(r f_{\lambda}\right)}{r f_{\lambda}}\right\}_{r=a ; \lambda=l}} \frac{f_{l}(b) f_{l}(r)}{f_{l}^{2}(a)} \frac{P_{l}\{\cos (\pi-\vartheta)\}}{\sin (\pi l)} .
$$

The constant $B$ represents the moment of the dipole. Each function $f_{l}(r)$ constitutes a solution of the height-gain differential equation, that has to satisfy the radiation condition at infinity. 


\section{Logarithmic Derivative of the Height-Gain Function at the Earth's Surface}

The boundary condition (23) can be put in the following form for the individual modes:

Hence the quantity

$$
\left\{\frac{\frac{\partial}{\partial r}\left(r f_{\lambda}\right)}{r f_{\lambda}}\right\}_{r=a ; \lambda=l}=\frac{\Gamma}{a} .
$$

$$
\left\{\frac{\frac{\partial}{\partial r}\left(r f_{\lambda}\right)}{r f_{\lambda}}\right\}_{r=a}
$$

proves to be important in connection with the evaluation of both the eigenvalues $l$, and of the denominator of the amplitude factor in (24). Its approximation for small values of $h / a$ and of $\delta C_{l}=C_{l}-n(a)$ (see the end of section 5 ) will be discussed now.

We start from the extended W.K.B. approximation (19) for $r f_{\lambda}$. Its logarithmic derivative becomes:

$$
\frac{\frac{\partial}{\partial r}\left(r f_{\lambda}\right)}{r f_{\lambda}}=\frac{1}{6} \frac{u_{\lambda}^{\prime}}{u_{\lambda}}-\frac{1}{2} \frac{u_{\lambda}^{\prime \prime}}{u_{\lambda}^{\prime}}-e^{-i \pi / 3} u_{\lambda}^{\prime} \frac{H_{2 / 3}^{(1)}\left(u_{\lambda}\right)}{H_{1 / 3}^{(1)}\left(u_{\lambda}\right)} .
$$

Another logarithmic derivation of the expression

$$
u_{\lambda}^{\prime}=\frac{k_{0} a}{r}\left\{M_{\text {eff }}^{2}(r)-C_{l}^{2}\right\}^{1 / 2} \sim k_{0}\left\{M_{\text {eff }}^{2}(r)-C_{l}^{2}\right\}^{1 / 2}
$$

results in:

$$
\frac{u_{\lambda}^{\prime \prime}}{u_{\lambda}^{\prime}} \sim \frac{M_{\mathrm{eff}}(r) \cdot M_{\mathrm{eff}}^{\prime}(r)}{M_{\mathrm{eff}}^{2}(r)-C_{l}^{2}} .
$$

We next introduce a definition for the effective-earth radius for any profile, viz:

$$
a_{\mathrm{eff}}=\left[\frac{r n_{\mathrm{eff}}(r)}{\frac{d}{d r}\left\{r n_{\mathrm{eff}}(r)\right\}}\right]_{r=a}=\frac{M_{\mathrm{eff}}(a)}{M_{\mathrm{eff}}^{\prime}(a)} .
$$

This definition involves the following value of (28) at $r=a$ :

$$
\left(\frac{u_{\lambda}^{\prime \prime}}{u_{\lambda}^{\prime}}\right)_{r=a} \sim \frac{n_{\mathrm{eff}}^{2}(a)}{a_{\mathrm{eff}}\left\{n_{\mathrm{eff}}^{2}(a)-C_{l}^{2}\right\}} .
$$

On the other hand, the expansion (21) may be approximated for small $\mathrm{h} / a$ and $\delta C_{l}$ by its first term. Its value for $r=a$ reduces to:

$$
u_{\lambda}(a) \sim \frac{k_{0} a_{\mathrm{eff}}}{3} \frac{\left\{n_{\mathrm{eff}}^{2}(a)-C_{\lambda}^{2}\right\}^{3 / 2}}{n_{\mathrm{eff}}^{2}(a)} .
$$

A comparison of (30), (31), and of the special value of (27) at $r=a$, then proves the vanishing (for the approximations under consideration) of the quantity

$$
\frac{1}{6} \frac{u_{\lambda}^{\prime}}{u_{\lambda}}-\frac{1}{2} \frac{u_{\lambda}^{\prime \prime}}{u_{\lambda}^{\prime}}
$$

at $r=a$. Further, when evaluating the remaining term in the right-hand side of (6) for $r=a$, we may substitute, applying (27) and (31)

$$
u_{\lambda}^{\prime}(a) \sim k_{0}\left\{n_{\text {eff }}^{2}(a)-C_{l}^{2}\right\}^{1 / 2} \sim k_{0}\left\{\frac{3 n_{\text {eff }}^{2}(a)}{k_{0} a_{\text {eff }}}\right\}^{1 / 3}\left\{u_{\lambda}(a)\right\}^{1 / 3} .
$$


The following final approximation thus results from (26) for $r=a$ :

$$
\left\{\frac{\frac{\partial}{\partial r}\left(r f_{\lambda}\right)}{r f_{\lambda}}\right\}_{r=a} \sim-e^{-i \pi / 3}\left\{\frac{3 k_{0}^{2} n_{\mathrm{eff}}^{2}(a)}{a_{\mathrm{eff}}}\right\}^{1 / 3} u_{\lambda}^{1 / 3}(a) \frac{H_{2 / 3}^{(1)}\left\{u_{\lambda}(a)\right\}}{H_{1 / 3}^{(1)}\left\{u_{\lambda}(a)\right\}} .
$$

\section{Equation for the Eigenvalues}

According to (5) and (33) the eigenvalues are to be determined from the equation:

$$
u_{l}^{1 / 3}(a) \frac{H_{2 / 3}^{(1)}\left\{u_{l}(a)\right\}}{H_{1 / 3}^{(1)}\left\{u_{i}(a)\right\}}=-\frac{\Gamma}{a} e^{i \pi / 3}\left\{\frac{a_{\mathrm{eff}}}{3 k_{0}^{2} n_{\mathrm{eff}}^{2}(a)}\right\}^{1 / 3} .
$$

The resulting roots $u_{l}(a)$ fix in succession the corresponding values of $C_{l}$ and $l$ (see (31) and (14)).

The only profile parameters entering in the equations (34), (31), and (14) for the eigenvalues are $n(a)$, and the effective earth's radius $a_{\text {eff }}$ defined by (29). Hence the conventional diffraction theory for a homogeneous atmosphere with refractive index $n(a)$ can be applied at once, provided that the actual earth's radius $a$ is replaced by $a_{\text {eff. }}$ This simple result depends on the smallness of $\delta C_{l}=C_{l}-n(a)$ which will be proved later on in the rigorous theory of part II. It then appears that the eigenvalues do only depend, in the approximations under consideration, on profile properties of the refractive index near the earth's surface; these properties concern $n(a)$ and the derivative $n^{\prime}(a)$, both of which determine the parameter $a_{\text {eff }}$. However, we emphasize the assumption of a smooth profile $n(r)$ without any discontinuity of a derivative of any order; such a discontinuity would involve higher-order terms in the expansion (21) for $u_{l}(r)$ which are not negligible with respect to the first term.

\section{Approximate Expansion in Terms of Modes}

In order to obtain a final approximation replacing (24) we have to evaluate, among others, the derivative of (33) with respect to $\lambda$. This can be facilitated with the aid of two relations, to be derived as follows. First we obtain from a differentiation of (31):

$$
\frac{\partial u_{\lambda}(a)}{\partial \lambda} \sim \frac{-3^{1 / 3}}{2}\left\{\frac{k_{0} a_{\text {eff }}}{n_{\text {eff }}^{2}(\mathrm{a})}\right\}^{2 / 3} u_{\lambda}^{1 / 3}(a) \frac{\partial\left(C_{\lambda}^{2}\right)}{\partial \lambda}
$$

The last factor can further be reduced, with the aid of the approximation $C_{l} \sim l / k_{0} a$ of (14). We find:

$$
\frac{\partial\left(C_{\lambda}^{2}\right)}{\partial \lambda}=\frac{2 \lambda+1}{k_{0}^{2} a^{2}} \sim \frac{2\{\lambda(\lambda+1)\}^{1 / 2}}{k_{0}^{2} a^{2}} \sim \frac{2 C_{\lambda}}{k_{0} a}
$$

On the other hand, we shall make use of the identity

$$
\frac{d}{d u}\left\{\frac{u^{1 / 3} H_{2 / 3}^{(1)}(u)}{H_{1 / 3}^{(1)}(u)}\right\}=e^{i \pi / 3} u^{1 / 3}\left[1+\left\{e^{-i r / 3} \frac{H_{2 / 3}^{(1)}(u)}{H_{1 / 3}^{(1)}(u)}\right\}^{2}\right] .
$$

The application of (35), (36) and (37) to the determination of the $\lambda$ derivative of (33) results in an expression which can further be simplified for $\lambda$ equaling an eigenvalue $l$. In fact, we then have $C_{\lambda}=C_{l} \sim n_{\text {eff }}(a)$; this latter zero-order approximation [which is equivalent to $\left.l \sim k_{0} a n_{\text {eff }}(a)\right]$ is well known from the diffraction theory for a homogeneous atmosphere. Moreover, we may then also eliminate the Hankel functions with the aid of (34). The procedure outlined here results in:

$$
\frac{\partial}{\partial \lambda}\left\{\frac{\frac{\partial}{\partial r}\left(r f_{\lambda}\right)}{r f_{\lambda}}\right\}_{r=a ; \lambda=l} \sim \frac{3^{2 / 3} k_{0}^{1 / 3} a_{\text {eff }}^{1 / 3} n_{\text {eff }}(a)}{a}\left[u_{l}^{2 / 3}(a)+\frac{\Gamma^{2}}{a^{2}}\left\{\frac{a_{\text {eff }}}{3 k_{0}^{2} n_{\text {eff }}^{2}(a)}\right\}^{2 / 3}\right] .
$$


We further substitute in (24):

$$
2 l+1 \sim 2 l \sim 2 k_{0} a n_{\text {eff }}(a)
$$

as well as the well-known approximation for Legendre functions with large complex order having a positive imaginary part. The latter reads:

$$
\frac{P_{l}\{\cos (\pi-\vartheta)\}}{\sin (\pi l)} \sim-(2 i)^{1 / 2} \frac{e^{i l \vartheta}}{(\pi l \sin \vartheta)^{1 / 2}} .
$$

In view of the approximate value $l \sim k_{0} a n_{\text {eff }}(a)$ we introduce the representation

$$
l=k_{0} a n_{\text {eff }}(a)+\delta l
$$

whereas the correction $\delta l$ may be omitted in the factor $l^{1 / 2}$. The substitution of (38), (39), (40), and (41) into (24) leads to:

$$
\Pi \sim-\frac{2^{3 / 2}(i \pi)^{1 / 2} B k_{0}^{1 / 6} e^{i k_{0} a n_{\mathrm{eff}}(a) \vartheta}}{c b a_{\mathrm{eff}}^{1 / 3}\left\{a n_{\mathrm{eff}}(a) \sin \vartheta\right\}^{1 / 2}} \sum_{l} \frac{f_{l}(b) f_{l}(r)}{f_{l}^{2}(a)}\left[\left\{3 u_{l}(a)\right\}^{2 / 3}+\frac{\Gamma^{2}}{a^{2}}\left\{\frac{a_{\mathrm{eff}}}{k_{0}^{2} n_{\mathrm{eff}}^{2}(a)}\right\}^{2 / 3}\right]
$$

We are interested first of all in the field strength attenuation that is due to the atmospheric refraction and the diffraction by the earth. Therefore, we next pass to the ratio of $|\Pi|$ and the modulus $\left|\Pi_{p r}\right|$ of the scalar determining the primary unattenuated field. The latter modulus reads [4]:

$$
\left|\Pi_{p r}\right|=\frac{B}{c b} \frac{1}{T P} \sim \frac{B}{c b a \vartheta}
$$

TP being the distance from the transmitter to the receiver.

We thus obtain from (42) and (43):

$$
\left|\frac{\Pi}{\Pi_{p r}}\right| \sim \frac{2^{3 / 2} k_{0}{ }^{1 / 6}}{a_{\mathrm{eff}}^{1 / 3}}\left\{\frac{\pi a \vartheta}{n_{\mathrm{eff}}(a)}\right\}^{1 / 2} \mid \sum_{l} \frac{f_{l}(b) f_{l}(r)}{f_{l}^{2}(a)}\left[\left\{3 u_{l}(a)\right\}^{2 / 3}+\frac{\Gamma^{2}}{a^{2}}\left\{\frac{a_{\mathrm{eff}}}{k_{0}^{2} n_{\mathrm{eff}}^{2}(a)}\right\}^{2 / 3}\right] .
$$

The effects of the elevations $b-a$ of the transmitter, and $r-a$ of the receiver, are contained, for each individual mode, in the height-gain factors

$$
\frac{f_{l}(b)}{f_{l}(a)} \text { and } \frac{f_{l}(r)}{f_{l}(a)} \text {. }
$$

These factors depend, according to (19) and (20), on the complete profile between the earth's surface and the levels of the transmitter and receiver. On the other hand, these factors can be left out of consideration when considering propagation along the ground. In this case the field is completely determined by $n_{\text {eff }}(a), a_{\text {eff }}$ and $\Gamma$; the dependence on the profile of the refractive index (if assumed as smooth), then only concerns its properties at the vicinity of the earth's surface.

\section{Connection of the Height-Gain Factors With Horizon Distances}

For an investigation of the height-gain effects we can restrict ourselves to an elevated receiver, the dependence on the height being completely similar for an elevated transmitter. Substitution of the extended W.K.B. approximation (19) in both $f_{l}(r)$ and $f_{l}(a)$ yields:

$$
\frac{f_{l}(r)}{f_{l}(a)} \sim \frac{a}{r}\left\{\frac{u_{l}(r) \cdot u_{l}^{\prime}(a)}{u_{l}(a) \cdot u_{l}^{\prime}(r)}\right\}^{1 / 2} \frac{H_{1 / 3}^{(1)}\left\{u_{l}(r)\right\}}{H_{1 / 3}^{(1)}\left\{u_{l}(a)\right\}} .
$$


The effect of the height $h=r-a$ enters implicitly through the variable $u_{l}(r)$, and also in the factor

$$
\frac{1}{r\left\{u_{l}^{\prime}(r)\right\}^{1 / 2}}=\frac{1}{\left(k_{0} a r\right)^{1 / 2}\left\{M_{\text {eff }}^{2}(r)-C_{l}^{2}\right\}^{1 / 4}} \cdot
$$

The dependence on the height can therefore be expressed by:

$$
\frac{f_{l}(r)}{f_{l}(a)} \sim \frac{F\left\{u_{l}(r)\right\}}{r^{1 / 2}\left\{M_{\mathrm{eff}}^{2}(r)-C_{l}^{2}\right\}^{1 / 4}} .
$$

The variable $u_{l}(r)$ can be connected with a geometric property of the profile, viz the angular distance $\vartheta(r)$ from the elevated receiver to its horizon point. This distance can be evaluated with the aid of Snell's law for the curved ray trajectory that passes through the receiver, and meets the earth tangentially. For our spherically symmetric medium this law amounts to the relation (11), if its refractive index is given by $n_{\text {eff }}(r)$ instead of $n(r)$ [thus differing slightly from the actual refractive index in the case of the electric solution, see (6)]. We have:

$$
r^{\prime} n_{\mathrm{eff}}\left(r^{\prime}\right) \sin \tau\left(r^{\prime}\right)=a n_{\mathrm{eff}}(a)
$$

if $\tau\left(r^{\prime}\right)$ represents the angle, at an arbitrary point $\left(r_{,}^{\prime}, \vartheta^{\prime}\right)$ of the trajectory, between the tangent of the latter and the vertical through this point. Hence $\tan \tau=r^{\prime} d \vartheta / d r^{\prime}$.

The relation (47), or the equivalent one

$$
M_{\text {eff }}\left(r^{\prime}\right) \sin \tau\left(r^{\prime}\right)=M_{\text {eff }}(a),
$$

can also be put in the form:

$$
\frac{d \vartheta^{\prime}}{d r^{\prime}}=\frac{\tan \tau\left(r^{\prime}\right)}{r^{\prime}}=\frac{M_{\mathrm{eff}}(a)}{r^{\prime}\left\{M_{\mathrm{eff}}^{2}\left(r^{\prime}\right)-M_{\mathrm{eff}}^{2}(a)\right\}^{1 / 2}} .
$$

An integration along the trajectory, from the horizon point at $r^{\prime}=a$ up to the receiver at $r^{\prime}=r$, yields the following formula for the angular horizon distance $\vartheta(r)$ of the receiver:

$$
\vartheta(r)=M_{\mathrm{eff}}(a) \int_{a}^{r} \frac{d r^{\prime}}{r^{\prime}\left\{M_{\mathrm{eff}}^{2}\left(r^{\prime}\right)-M_{\mathrm{eff}}^{2}(a)\right\}^{1 / 2}} \cdot
$$

We also need the derivative of this function, viz

$$
\frac{d \vartheta}{d r}=\frac{M_{\mathrm{eff}}(a)}{r\left\{M_{\mathrm{eff}}^{2}(r)-M_{\mathrm{eff}}^{2}(a)\right\}^{1 / 2}} \cdot
$$

The connection between $r$ and $\vartheta(r)$ is unique for the smooth troposphere profile (excluding, e.g., "ducts") under consideration. Therefore, all functions of $r$ may also be considered as functions of $\vartheta(r)$, in particular the variable $u_{l}(r)$. In order to determine the explicit dependence of $u_{l}(r)$ on $\vartheta(r)$, we first derive, with the aid of (49), the relation:

$$
\frac{d\left\{M_{\mathrm{eff}}^{2}(r)-M_{\mathrm{eff}}^{2}(a)\right\}^{1 / 2}}{d \vartheta}=\frac{r \frac{d\left(M^{2}\right)}{d r}}{2 n(a)} .
$$

We can deduce similar relations for the higher-order derivatives of $\left\{M_{\text {eff }}^{2}(r)-M_{\text {eff }}^{2}(a)\right\}^{1 / 2}$ with respect to $\vartheta$, again using (49); for instance we find:

$$
\frac{d^{2}}{d \vartheta^{2}}\left\{M_{\mathrm{eff}}^{2}(r)-M_{\mathrm{eff}}^{2}(a)\right\}^{1 / 2}=\frac{r}{2 M_{\mathrm{eff}}^{2}(a)} \cdot \frac{d}{d r}\left\{r \frac{d M_{\mathrm{eff}}^{2}(r)}{d r}\right\} \cdot\left\{M_{\mathrm{eff}}^{2}(r)-M_{\mathrm{eff}}^{2}(a)\right\}^{1 / 2}
$$

The special values of these derivatives at $r=a$ can be expressed in terms of the coefficients $\Lambda_{j}$ defined by (17). These latter derivatives determine the coefficients of the Taylor expansion 
of $\left\{M_{\text {eff }}^{2}(r)-M_{\text {eff }}^{2}(a)\right\}^{1 / 2}$, with respect to $\vartheta$, taken at the point $r=a$, that is $\vartheta(r)=0$. The expansion in question starts as follows:

$$
M_{\text {eff }}^{2}(r)=M_{\text {eff }}^{2}(a)+\frac{\Lambda_{1}^{2}}{4 n_{\mathrm{eff}}^{2}(a)} \vartheta^{2}(r)+\frac{\Lambda_{1}^{2}\left(\Lambda_{1}+\Lambda_{2}\right)}{24 n_{\mathrm{eff}}^{4}(a)} \vartheta^{4}(r)+\ldots ;
$$

moreover, the definition (29) implies the relation

$$
\Lambda_{1}=2 n_{\text {eff }}^{2}(a) \frac{a}{a_{\text {eff }}} .
$$

We next consider the approximation of $u_{l}(r)$ by the first term of (21), viz

$$
u_{l}(r) \sim \frac{2}{3} k_{0} a \frac{\left\{M_{\mathrm{eff}}^{2}(r)-C_{l}^{2}\right\}^{3 / 2}}{r \frac{d\left(M_{\mathrm{eff}}^{2}\right)}{d r}}=\frac{2}{3} \frac{k_{0} a}{n_{\mathrm{eff}}(a)} \frac{\left\{M_{\mathrm{eff}}^{2}(r)-C_{l}^{2}\right\}^{3 / 2}\left\{M_{\mathrm{eff}}^{2}(r)-M_{\mathrm{eff}}^{2}(a)\right\}^{1 / 2}}{d\left(M_{\mathrm{eff}}^{2}\right) / d \vartheta}
$$

Each factor can be expanded with the aid of (50), but we shall only retain terms up to the first one depending on $\vartheta$. This requires the validity of the relation

$$
\left(\Lambda_{1}+\Lambda_{2}\right) \cdot \vartheta^{2}(r) \ll n^{2}(a) .
$$

Moreover we substitute [compare (14) and (41)]:

$$
C_{l}^{2}=\left\{n_{\mathrm{eff}}(a)+\delta C_{l}\right\}^{2} \sim n_{\mathrm{eff}}^{2}(a)+2 n_{\mathrm{eff}}(a) \cdot \delta C_{l} \sim n_{\mathrm{eff}}^{2}(a)+2 n_{\mathrm{eff}}(a) \frac{\delta l}{k_{0} a} .
$$

The corresponding evaluation of (52) results in:

$$
u_{l}(r) \sim \frac{k_{0} a_{\mathrm{eff}}}{3\left\{n_{\mathrm{eff}}(a)\right\}^{1 / 2}} \cdot\left\{-\frac{2 \delta l}{k_{0} a}+n_{\mathrm{eff}}(a) \frac{a^{2}}{a_{\mathrm{eff}}^{2}} \cdot \vartheta^{2}(r)\right\}^{3 / 2} .
$$

Apart from the inequality (53) this approximation depends on the neglect of the higherorder terms of (21). This neglect proves to be justified in view of the smallness of $\delta l / k_{0} a$ and $\vartheta^{2}$ compared to unity, remembering also the order of magnitude of unity for $n_{\text {eff }}(a)$ and $a / a_{\text {eff }}$. Moreover, (53) is even satisfied automatically for all $\vartheta(r)<<1$ provided that we have $\left|\Lambda_{2}\right|<<\Lambda_{1}$. Under such circumstances $u_{l}(r)$ only depends on the eigenvalue $\delta l$, the horizon distance $\vartheta(r)$, and the profile properties $n_{\text {eff }}(a)$ and $a_{\text {eff }}$; on the other hand, the eigenvalues $\delta l$ merely depend on $n_{\text {eff }}(a)$ and $a_{\text {eff }}$ (see section 8). As a consequence $n_{\text {eff }}(a), a_{\text {eff }}$ and $\vartheta(r)$ may be considered as the only quantities determining $u_{l}(r)$. The same then holds for $F\left\{u_{l}(r)\right\}$ in (46), as well as for the quantity [compare (50) and (54)],

$$
\left\{M_{\mathrm{eff}}^{2}(r)-C_{l}^{2}\right\}^{1 / 4}=\left\{-2 n_{\mathrm{eff}}(a) \frac{\delta l}{k_{0} a}+\frac{\Lambda_{1}^{2}}{4 n_{\mathrm{eff}}^{2}(a)} \vartheta^{2}(r)\right\}^{1 / 4} .
$$

The still remaining factor $r^{-1 / 2}$ of (46) can be approximated by $a^{-1 / 2}$. We thus finally conclude that the height-gain factor does depend, in the approximations under consideration [the most restrictive one being (53)], on no other quantities than $n_{\text {eff }}(a), a_{\text {eff }}$ and $\vartheta(r)$.

\section{Asymptotic Approximation of the Height-Gain Factors}

We might substitute the approximations of the preceding section into the height-gain factors (45) for both the transmitter and the receiver, and next the values of the latter into the mode expansion (44). This would show the explicit dependence of the attenuation factor $\left|\Pi / \Pi_{p r}\right|$ on the four quantities $n_{\text {eff }}(a), a_{\text {eff }}, \vartheta\left(r_{1}\right)$ and $\vartheta\left(r_{2}\right) ; \vartheta\left(r_{1}\right)$ and $\vartheta\left(r_{2}\right)$ here represent the angular distances from the transmitter and receiver to their horizon point. The complicated final expression depends, among other things, on Hankel functions of order $1 / 3$. The latter can be simplified considerably by replacing them by their asymptotic approximation. This can be justified in the case of an argument with modulus well above unity. Unfortunately, the argument $u_{l}(a)$ of the Hankel functions in the denominator of (45) proves to be of the order of unity, but the other argument $u_{l}(r)$ of the Hankel functions in the numerator of $(45)$ may be much larger for elevated transmitters and receivers. 
Let us consider, once again, the height-gain function for the receiver, and estimate the order of magnitude of $\left|u_{l}(r)\right|$. When leaving out of consideration the term with $\vartheta^{2}$ in (55) we find an order of unity for $\left|u_{l}(r)\right|$, remembering (from the case of a homogeneous atmosphere) the order of $\left(k_{0} a\right)^{1 / 3}$ for $\delta l$. Therefore, $\left|u_{l}(r)\right|>>1$ will certainly hold provided we have

$$
n_{\mathrm{eff}}(a) \frac{a^{2}}{a_{\mathrm{eff}}^{2}} \vartheta^{2}(r) \gg \frac{|2 \delta l|}{k_{0} a} \approx \frac{1}{\left(k_{0} a\right)^{2 / 3}} .
$$

Hence, a sufficient condition for applying the asymptotic expression for $H_{1 / 3}^{(1)}\left\{u_{l}(r)\right\}$ reads very roughly $\left[n_{\text {eff }}(a)\right.$ and $a / a_{\text {efr }}$ being of the order of unity]:

$$
\vartheta(r) \gg \frac{1}{\left(k_{0} a\right)^{1 / 3}} .
$$

This inequality holds for altitudes $h=r-a$ above some critical value, $h_{c r}$ say.

Let us now assume $h>h_{c r}$ for the receiver in question. We may then also replace the expression between braces in (55) by the first two terms of its binomial expansion with respect to $\delta l$, so as to obtain:

$$
u_{l}(r) \sim \frac{k_{0} n_{\text {eff }}(a) a^{3}}{3 a_{\text {eff }}^{2}} \vartheta^{3}(r)-\delta l \cdot \vartheta(r) .
$$

The substitution of the asymptotic expression for $H_{1 / 3}^{(1)}\left\{u_{l}(r)\right\}$ into (44) first results in:

$$
\frac{f_{l}(r)}{f_{l}(a)} \sim \frac{a}{r}\left\{\frac{2}{\pi} \frac{u_{l}^{\prime}(a)}{u_{l}(a) \cdot u_{l}^{\prime}(r)}\right\}^{1 / 2} \frac{\left.e^{i\left\{u_{l}(r)-\frac{5}{12} \pi\right.}\right\}}{H_{1 / 3}^{(1)}\left\{u_{l}(a)\right\}} .
$$

In the exponent we must take into account both terms of (58), but the dominating first term suffices in the nonexponential factors. We shall substitute, moreover, the relations (31) and (32) for evaluating $u_{l}(a)$ and $u_{l}{ }^{\prime}(a)$ [applying also (54)], the approximation $a / r \sim 1$, and finally the following relation based on $(20),(50),(54),(51)$ and (56):

$$
\begin{aligned}
u_{l}^{\prime}(r)=\frac{k_{0} a}{r}\left\{M_{\mathrm{eff}}^{2}(r)-C_{l}^{2}\right\}^{1 / 2} \sim k_{0}\left\{M_{\mathrm{eff}}^{2}(a)-C_{l}^{2}+\frac{\Lambda_{1}^{2}}{4 n_{\mathrm{eff}}^{2}(a)} \vartheta^{2}(r)\right\}^{1 / 2} \\
\quad=k_{0}\left\{-2 n_{\mathrm{eff}}(a) \frac{\delta l}{k_{0} a}+\frac{n_{\mathrm{eff}}^{2}(a) a^{2}}{a_{\mathrm{eff}}^{2}} \vartheta^{2}(r)\right\}^{1 / 2} \sim k_{0} n_{\mathrm{eff}}(a) \frac{a}{a_{\mathrm{eff}}} \vartheta(r) .
\end{aligned}
$$

We then find:

$$
\frac{f_{l}(r)}{f_{l}(a)} \sim\left(\frac{6}{\pi}\right)^{1 / 2} \frac{e^{i\left\{\frac{k_{0} n_{\mathrm{eff}}(a) a^{3}}{3 a_{\mathrm{eff}}^{2}} \cdot \vartheta^{3}(r)-\delta l \cdot \vartheta(r)-\frac{5}{12} \pi\right\}}}{\{-2 \delta l \cdot \vartheta(r)\}^{1 / 2} \cdot H_{1 / 3}^{(1)}\left[\frac{a_{\mathrm{eff}}(-2 \delta l)^{3 / 2}}{3\left\{k_{0} n_{\mathrm{eff}}(a)\right\}^{1 / 2} a^{3 / 2}}\right]} .
$$

\section{Mode Expansion for Transmitter and (or) Receiver Above the Critical Altitude}

We first assume a receiver at an elevation $h_{2}=r_{2}-a$ above the critical height $h_{c r}$ given by (57), and a transmitter on the ground. When evaluating the mode expansion (44) we can then substitute (59) for the height-gain factor of the receiver, that of the transmitter being unity. The term proportional to $\vartheta^{3}$ in the exponent of (59) drops out, it being real and independent of $l$; it therefore has no effect on the modulus $\left|\Pi / \Pi_{p r}\right|$.

The use of this substitution results in:

$$
\left|\frac{\Pi}{\Pi_{p r}}\right| \sim \frac{2^{3 / 2} k_{0}^{1 / 6}}{a_{\mathrm{eff}}^{1 / 3}}\left\{\frac{3 a \vartheta}{n_{\mathrm{eff}}(a) \vartheta\left(r_{2}\right)}\right\}^{1 / 2} \times\left|\sum_{l} \frac{e^{i \delta l\left\{\vartheta-\vartheta\left(r_{2}\right)\right\}}}{(\delta l)^{1 / 2}\left[\left\{3 u_{l}(a)\right\}^{2 / 3}+\frac{\Gamma^{2}}{a^{2}}\left\{\frac{a_{\mathrm{eff}}}{k_{0}^{2} n_{\mathrm{eff}}^{2}(a)}\right\}^{2 / 3}\right] \cdot H_{1 / 3}^{(1)}\left\{u_{l}(a)\right\}}\right|,
$$


in which

$$
u_{l}(a)=\frac{a_{\mathrm{eff}}(-2 \delta l)^{3 / 2}}{3\left\{k_{0} n_{\mathrm{eff}}(a)\right\}^{1 / 2} a^{3 / 2}} \cdot
$$

We next consider a transmitter and receiver at elevations $h_{1}=r_{1}-a$ and $h_{2}=r_{2}-a$ which are both above the critical height. We may then apply (59) to each of the two height-gain factors $f_{l}\left(r_{1}\right) / f_{l}(a)$ and $f_{l}\left(r_{2}\right) / f_{l}(a)$ occurring in (44). It leads to the expression:

$$
\left|\frac{\Pi}{\Pi_{p r}}\right| \sim \frac{6 k_{0}^{1 / 6}}{a_{\mathrm{eff}}^{1 / 3}} \frac{(2 a \vartheta)^{1 / 2}}{\left\{\pi n_{\mathrm{eff}}(a) \vartheta\left(r_{1}\right) \vartheta\left(r_{2}\right)\right\}^{1 / 2}} \times\left|\sum_{l} \frac{e^{i \delta l\left\{\vartheta-\vartheta\left(r_{1}\right)-\vartheta\left(r_{2}\right)\right\}}}{\delta l\left[\left\{3 u_{l}(a)\right\}^{2 / 3}+\frac{\Gamma^{2}}{a^{2}}\left\{\frac{a_{\mathrm{eff}}}{k_{0}^{2} n_{\mathrm{eff}}^{2}(a)}\right\}^{2 / 3}\right] \cdot\left[H_{1 / 3}^{(1)}\left\{u_{l}(a)\right\}\right]^{2} \mid}\right|
$$

\section{Final Expressions for the Vertical Field Component}

So far we have derived expressions for the scalar quantity $\Pi$. The transition to the field components is conditioned by (3) and (4). In the case of the electric solution this transition is effected for the vertical component $E_{r}$ of the electric field by multiplying the coefficient of each individual mode by $[5] i l(l+1) /\left(k_{0} r\right)$. In view of our approximations this quantity can be simplified to

$$
\frac{i l^{2}}{k_{0} a} \sim i k_{0} a n_{\mathrm{eff}}^{2}(a)
$$

By combining this quantity with the expression (43), that is

$$
\left|\Pi_{p r}\right| \sim \frac{B}{c b a \vartheta} \sim \frac{B}{c a^{2} \vartheta}
$$

we deduce the following converting factor for passing from the attenuation factor $\left|\Pi / \Pi_{p r}\right|$ to $\left|E_{r}\right|$ :

$$
k_{0} a \cdot n_{\mathrm{eff}}^{2}(a) \frac{B}{c a^{2} \vartheta}=\frac{k_{0} B n_{\mathrm{eff}}^{2}(a)}{c a \vartheta}
$$

A similar factor proportional to $\vartheta^{-1}$ applies to the reduction of $\left|\Pi / \Pi_{p r}\right|$ to the vertical magnetic field component $\left|H_{r}\right|$ in the case of the magnetic solution.

With the aid of this factor we find the following representation replacing (44):

$$
\left|E_{r}\right| \sim \frac{2^{3 / 2} k_{0}^{7 / 6} B n_{\mathrm{eff}}^{3 / 2}(a)}{c a_{\mathrm{eff}}^{1 / 3}}\left(\frac{\pi}{a \vartheta}\right)^{1 / 2} \times \mid \sum_{l} \frac{f_{l}(b) f_{l}(r)}{f_{l}^{2}(a)}\left[\left\{3 u_{l}(a\}^{2 / 3}+\frac{\Gamma^{2}}{a^{2}}\left\{\frac{a_{\mathrm{eff}}}{k_{0}^{2} n_{\mathrm{eff}}^{2}(a)}\right\}^{2 / 3}\right]\right.
$$

We also mention the field representations corresponding to (60) and (61) for a transmitter and (or) receiver well above the critical height:

$$
\begin{aligned}
& \left|E_{r}\right| \sim^{2^{3 / 2} k_{0}^{7 / 6} B \eta_{\text {eff }}^{3 / 2}(a)}=\frac{3}{c a_{\text {eff }}^{1 / 3}}\left\{\frac{e^{i \delta l}\left\{\vartheta-\vartheta\left(r_{2}\right)\right\}}{a \vartheta \cdot \vartheta\left(r_{2}\right)}\right\}^{1 / 2} \times\left|\sum_{l} \frac{\Gamma^{2}}{(\delta l)^{1 / 2}\left[\left\{3 u_{l}(a)\right\}^{2 / 3}+\frac{\Gamma^{2}}{a^{2}}\left\{\frac{a_{\text {eff }}}{k_{0}^{2} n_{\text {eff }}^{2}(a)}\right\}^{2 / 3}\right] \cdot H_{\frac{1}{3}\{}^{(1)}\left\{u_{l}(a)\right\}}\right|, \\
& \left(h_{1}=0 ; h_{2}>h_{c r}\right)
\end{aligned}
$$

and

$$
\begin{gathered}
|E r| \sim \frac{6 k_{0}^{7 / 6} B n_{\text {eff }}^{3 / 2}(a)}{c a_{\text {eff }}^{1 / 3}}\left\{\frac{2}{\pi a \vartheta \cdot \vartheta\left(r_{1}\right) \cdot \vartheta\left(r_{2}\right)}\right\}^{1 / 2} \times \mid \sum_{l} \frac{e^{i \delta l\left\{\vartheta-\vartheta\left(r_{1}\right)-\vartheta\left(r_{2}\right)\right\}}}{\delta l\left[\left\{3 u_{l}(a)\right\}^{2 / 3}+\frac{\Gamma^{2}}{a^{2}}\left\{\frac{a_{\text {eff }}}{k_{0}^{2} n_{\text {eff }}^{2}(a)}\right\}^{2 / 3}\right] \cdot\left[H_{\left.\frac{1}{3}(1)\left\{u_{l}(a)\right\}\right]^{2}}^{2} \mid\right.} . \\
\left(h_{1}>h_{c r} ; h_{2}>h_{c r}\right)
\end{gathered}
$$


All these expressions contain the quantity $H\left({ }_{1 / 3}^{(1)}\left\{u_{l}(a)\right\}\right.$ either explicitly, or implicitly in the height-gain factors. This quantity can not be reduced any further for the most general soil conditions of the earth. However, a convenient expression may be obtained in the two limiting cases of very long and very short waves. The latter case corresponds to an infinite value of the right-hand side of (34). Let us denote this right-hand side by $-e^{i \pi / 3} N$. The solution of (34) can then be expanded with respect to $N^{-1}$, a method well known in the diffraction theory for a homogeneous atmosphere [6]. A corresponding expansion then exists for $H_{1 / 3}^{(1)}\left\{u_{l}(a)\right\}$. Its leading term for large $|N|$ reads:

$$
H_{1 / 3}^{(1)}\left\{u_{l}(a)\right\} \sim-\frac{e^{-i \pi / 3} H_{2 / 3}^{(1)}\left(u_{l, \infty}\right) \cdot u_{l, \infty}^{1 / 3}}{\mathrm{~N}} ;
$$

$u_{l, \infty}$ here marks a zero of $H_{1 / 3}^{(1)}(u)$.

\section{Comparison Between the Fields Corresponding to Two Different Profiles}

As stated above (end of section 9), the influence of the refractive-index profile is restricted to that on the parameters $n_{\text {eff }}(a)$ and $a_{\text {eff }}$ for propagation over the ground. Moreover, the height-gain effects reduce to an additional dependence on the angular horizon distances $\vartheta\left(r_{1}\right)$ and $\vartheta\left(r_{2}\right)$ of the elevated transmitter and receiver (see section 10). This involves an extremely simple relation between the fields corresponding to two profiles having identical values of $n_{\text {eff }}(a)$ and $a_{\text {eff }}$. Such two profiles show the same surface values of the refractive index and its gradient, but they may differ noticeably at high altitudes. According to section 8 the eigenvalues $l$, too, prove to be identical for these profiles; therefore, the same then holds for all parameters occurring in expansions such as (62), (63) and (64).

We shall compare the actual profile with some reference profile, the quantities of which will be marked by a dash (see fig. 1 in which the Eckersley profile (22) is assumed to be the reference profile); both profiles are assumed to have coincident values of $a$ and $a_{\text {effi }}$. In view of the above remarks, raising of an antenna (transmitter or receiver) to an elevation $h=r-a$. will produce the same field as an elevated antenna at a height $h^{\prime}=r^{\prime}-a$ in the reference profile, provided that we have $\vartheta(r)=\vartheta^{\prime}\left(r^{\prime}\right)$.

This may be applied to atlases of propagation curves, such as edited by the C.C.I.R. [7] and by the Japanese Ministry of Postal Services [8]. The data of these atlases are essentially based on the Eckersley profile (22) with $a_{\text {eff }}=(4 / 3) a$. The evaluation of (48) for the angular horizon distance results for this profile in the expression:

$$
\vartheta_{\mathrm{Eck}}(r)=\left(\frac{a_{\mathrm{eff}}}{a}\right)^{1 / 2} \arccos \left(\frac{a}{r}\right) .
$$

Therefore, the curves of the mentioned atlases can also be used for other profiles with the same value of $a_{\text {eff }}$ the value of $n_{\text {eff }}(a)$ is irrelevant, since $\left.n_{\text {eff }}(a) \sim 1\right]$. The antenna heights $h=r-a$, corresponding to a horizon distance $\vartheta(r)$, are then to be replaced by an effective height $h^{\prime}=r^{\prime}-a$ for which

$$
r^{\prime}=\frac{a}{\cos \left\{\left(\frac{a}{a_{\text {eff }}}\right)^{1 / 2} \cdot \vartheta(r)\right\}}
$$

Examples of the use of such corrections are given in two recent publications by Norton $[9,10]$.

\section{Concept of the Effective or "Angular" Distance}

The most striking feature of the expressions (63) and (64) concerns the presence of the angular distances $\vartheta-\vartheta\left(r_{2}\right)$ and $\vartheta-\vartheta\left(r_{1}\right)-\vartheta\left(r_{2}\right)$ in the exponent. The effect of the antenna heights is mainly contained in the corresponding exponential, the influence of the nonexponential factors $\left\{\vartheta\left(r_{1}\right)\right\}^{1 / 2}$ and $\left\{\vartheta\left(r_{2}\right)\right\}^{1 / 2}$ being much smaller. The importance of the distances in 
question has been emphasized in particular by Norton, Rice, and Vogler [11]. These authors have introduced the term "angular distance" with an extension to the cases of propagation over irregular terrain. For propagation over a smooth spherical earth beyond the line of sight the "angular distance" represents simply the actual distance between the radio horizons of the transmitter and the receiver divided by the radius of the sphere.

For a smooth earth the expansions (63) and (64) justify the reduction of the height dependence to that on the "angular" distance, under the following conditions: (a) the elevated antennas are to be situated above the critical height $h_{c r}$ fixed by (57); and (b) the effect of the square roots $\left\{\vartheta\left(r_{1}\right)\right\}^{1 / 2}$ and $\left\{\vartheta\left(r_{2}\right)\right\}^{1 / 2}$ should be negligible.

Let us abstract from the factor $\vartheta^{1 / 2}$ in (63) and (64) which is not very critical. The dependence on the "angular distance" then combines both the influence of the antenna elevations and of the horizontal distance. The influence of the former simply amounts to a reduction of the actual distance $d=a \vartheta$ to that of the radio horizons, that is to $a\left\{\vartheta-\vartheta\left(r_{1}\right)\right\}$ or $a\left\{\vartheta-\vartheta\left(r_{1}\right)-\right.$ $\left.\vartheta\left(r_{2}\right)\right\}$. The comparison with a reference profile (see the preceding section) can be expressed here in terms of a shift $\Delta \vartheta$ of the "angular" distance. In fact, let an antenna height $h=r-a$ in the actual profile produce the same field, at a distance $d=a \vartheta$, as the reference profile at a distance $d+\Delta=a(\vartheta+\Delta \vartheta)$, the antenna elevation being kept constant. The unique dependence on the "angular" distance, assumed here for both profiles, then involves the relation:

Hence

$$
\vartheta-\vartheta(r)=\vartheta+\Delta \vartheta-\vartheta^{\prime}(r) .
$$

$$
\Delta \vartheta=\vartheta^{\prime}(r)-\vartheta(r)
$$

The corresponding linear distance shift is given, according to (48), by

$$
\Delta=a \Delta \vartheta=a M_{\mathrm{eff}}(a) \int_{a}^{r} \frac{d r^{\prime}}{r^{\prime}}\left[\frac{1}{\left\{M_{\mathrm{effi}}^{\prime 2}\left(r^{\prime}\right)-M_{\mathrm{eff}}^{2}(a)\right\}^{1 / 2}}-\frac{1}{\left\{M_{\mathrm{eff}}^{2}\left(r^{\prime}\right)-M_{\mathrm{eff}}^{2}(a)\right\}^{1 / 2}}\right] .
$$

The derivative with respect to the antenna elevation $h=r-a$, reading

$$
\frac{d \Delta}{d h} \sim M_{\mathrm{eff}}(a)\left[\frac{1}{\left\{M_{\mathrm{eff}}^{\prime 2}(r)-M_{\mathrm{eff}}^{2}(a)\right\}^{1 / 2}}-\frac{1}{\left\{M_{\mathrm{eff}}^{2}(r)-M_{\mathrm{eff}}^{2}(a)\right\}^{1 / 2}}\right]
$$

can be reduced for small altitudes with the aid of the expansions:

$$
\begin{aligned}
& M_{\mathrm{eff}}^{2}(r)-M_{\mathrm{eff}}^{2}(a)=\frac{\Lambda_{1}}{a} h+\frac{\Lambda_{2}}{2 a^{2}} h^{2}+\ldots, \\
& M_{\mathrm{eff}}^{\prime 2}(r)-M_{\mathrm{eff}}^{2}(a)=\frac{\Lambda_{1}}{a} h+\frac{\Lambda_{2}^{\prime}}{2 a^{2}} h^{2}+\ldots
\end{aligned}
$$

This leads to the following approximation for $\Delta$ itself for small heights:

$$
\Delta \sim \frac{n_{\text {eff }}(a)\left(\Lambda_{2}-\Lambda_{2}^{\prime}\right)}{6 a^{1 / 2} \Lambda_{1}^{3 / 2}} h^{3 / 2}=\frac{\left(\Lambda_{2}-\Lambda_{2}^{\prime}\right) a_{\mathrm{eff}}^{3 / 2}}{12.2^{1 / 2} n_{\mathrm{eff}}^{2}(a) a^{2}} h^{3 / 2}
$$

Equivalent expressions have been derived by Millington [12] and Wait [13] in a simplified derivation of the effective-distance concept.

\section{References and Notes}

[1] C. L. Pekeris, Asymptotic solutions for the normal modes in the theory of microwave propagation, J. Appl. Phys. 17, 1108 (1946).

[2] J. R. Wait, Radiation from a vertical antenna over a curved stratified ground, J. Research NBS $\mathbf{5 6}, 237$ (1956); see eq (6).

[3] H. Bremmer, Mode expansion in the low-frequency range for propagation through a curved stratified atmosphere, J. Research NBS 63D, 75 (1959); see eq (15). 
[4] See reference [3], eq (5)

[5] This follows from a comparison of the amplitude factors of the expansions (15) and (16) in reference [3].

[6] Compare H. Bremmer, Terrestrial radio waves, p. 45 (Elsevier Publishing Co., New York, N.Y., 1949) the quantities $\mu_{l}(a)$ and $(\Gamma / a)\left\{a_{e f f} / 3 k_{0}^{2} n_{e f f}^{2}(a)\right\}^{1 / 3}$ of the present paper correspond to $\left(-2 \tau_{s}\right)^{3 / 2} / 3$ and $1 /\left(3^{1 / 3} \delta\right)$ in this reference.

[7] Atlas of ground-wave propagation curves for frequencies between $30 \mathrm{Mc} / \mathrm{s}$ and $300 \mathrm{Mc} / \mathrm{s}$, published by the International Telecommunications Union, Geneva, Switzerland (1955).

[8] Atlas of radio wave propagation curves for frequencies between 30 and 10,000 Mc/s, prepared by the Radio Research Laboratory, Tokyo, Japan (January 1958).

[9] K. A. Norton, Transmission loss in radio propagation-II, NBS Tech. Note 12 (1959).

[10] K. A. Norton, System loss in radio wave propagation, J. Research NBS 63D, 53 (1959).

[11] K. A. Norton, P. L. Rice, and L. E. Vogler. The use of angular distance in estimating transmission loss and fading range for propagation through a turbulent atmosphere over irregular terrain, Proc. IRE 43, 1488 (1955). See in particular p. 1491.

[12] G. Millington, Propagation at great heights in the atmosphere, Marconi Rev. 21, 143 (1958).

[13] J. R. Wait, On the propagation of radio waves in an inhomogeneous atmosphere, NBS Tech. Note 2 (1959).

(Paper 64D5-82) 